Leukemia (2019) 33:2111-2115

https://doi.org/10.1038/s41375-019-0444-6

Chronic lymphocytic leukemia

\title{
$K R A S, N R A S$, and BRAF mutations are highly enriched in trisomy 12 chronic lymphocytic leukemia and are associated with shorter treatment-free survival
}

\author{
Elena Vendramini ${ }^{1} \cdot$ Riccardo Bomben $^{1} \cdot$ Federico Pozzo $^{1}$ - Dania Benedetti ${ }^{1}$. Tamara Bittolo ${ }^{1}$. \\ Francesca Maria Rossi ${ }^{1} \cdot$ Michele Dal Bo ${ }^{1}$ Kari G. Rabe ${ }^{2}$. Gabriele Pozzato ${ }^{3}$. Francesco Zaja ${ }^{3}$. Annalisa Chiarenza ${ }^{4}$. \\ Francesco Di Raimondo ${ }^{4}$ Esteban Braggio ${ }^{5} \cdot$ Sameer A. Parikh ${ }^{5} \cdot$ Neil E. Kay ${ }^{5} \cdot$ Tait D. Shanafelt $^{6}$. \\ Giovanni Del Poeta ${ }^{7} \cdot$ Valter Gattei $^{1} \cdot$ Antonella Zucchetto $^{1}$
}

Received: 4 March 2019 / Accepted: 6 March 2019 / Published online: 14 March 2019

(c) The Author(s) 2019. This article is published with open access

\section{To the Editor:}

$K R A S$ mutations are among the most common oncogenic events in human carcinomas of endodermal origin, whose presence predicts for resistance to several target therapies [1]. Conversely, little is known regarding the role and/or clinical impact of KRAS mutations in the setting of the hematological malignancies, including chronic lymphocytic leukemia (CLL), and only in recent years extensive sequencing data have highlighted the recurrent mutations of genes affecting the Ras-MAPK pathway in CLL [2, 3]. These mutations, by leading to a constitutive activation of MAPK signaling pathway, have emerged as relevant in driving impaired clinical responses to lenalidomide and chlorambucil, and acquired resistance to fludarabine as well as to PI3K and BCL2 inhibitors [4-6]. In this context, some studies pinpointed a higher frequency of mutations in

These authors contributed equally: Valter Gattei, Antonella Zucchetto

Supplementary information The online version of this article (https:// doi.org/10.1038/s41375-019-0444-6) contains supplementary material, which is available to authorized users.

Valter Gattei

vgattei@cro.it

$\triangle$ Antonella Zucchetto

zucchetto.soecs@cro.it

1 Clinical and Experimental Onco-Hematology Unit, Centro di Riferimento Oncologico di Aviano (CRO) IRCCS, Aviano (PN), Italy

2 Division of Biomedical Statistics and Informatics, Department of Health Sciences Research, Mayo Clinic, Rochester, MN, USA members of the Ras-MAPK pathway in CLL cases with specific clinico-biological features [6, 7], including the presence of trisomy 12 , a cytogenetic aberration associated with a unique pathophysiology among CLL [8, 9], and/or an unmutated (UM) configuration of IGHV genes, although a dedicated and comprehensive analysis of these aspects is still missing.

This study, approved by the IRB of the Aviano Centro di Riferimento Oncologico (Approvals n. IRB-05-2010 and n. IRB-05-2015), included 534 primary CLL from treatmentnaive patients. The cohort was purposely enriched in trisomy 12 CLL by including 110 cases from the Mayo Clinic, Rochester, MN [8] to better evaluate the incidence of mutations of the Ras-MAPK pathway in these subsets. Overall, out of 534 cases, trisomy 12 CLL accounted for 300 cases (190 with trisomy 12 as the sole abnormality [trisomy 12-only], and 110 with trisomy 12 plus another abnormality on FISH [trisomy 12-plus]), 332 cases had UM IGHV genes, and 214 cases had NOTCH1 aberrations (details in Table S1). CLL patients were diagnosed and treated according to the current iwCLL 2018 guidelines [10], and all samples were collected at diagnosis from treatment-naive patients. In 442/534 cases (clinical cohort),

3 Department of Internal Medicine and Haematology, Maggiore General Hospital, University of Trieste, Trieste, Italy

4 Division of Hematology, Ferrarotto Hospital, Catania, Italy

5 Division of Hematology, Department of Medicine, Mayo Clinic, Rochester, MN, USA

6 Department of Hematology/Oncology, Stanford University, Stanford, CA, USA

7 Division of Hematology, S. Eugenio Hospital and University of Tor Vergata, Rome, Italy 
treatment-free survival (TFS) data were available along with a comprehensive clinical and biological characterization (Table S1 and Supplemental Methods). This cohort showed the expected clinical behavior according to both the stratification of the established cytogenetic categories and to the canonical prognosticators by univariable and multivariable analyses (Supplemental Figure S1 and Table S2). Mutation testing for KRAS, NRAS, BRAF, TP53, NOTCH1, BIRC3, and $S F 3 B 1$ was performed on DNA from $\mathrm{CD} 19^{+}$enriched CLL samples by Next Generation Sequencing (NGS) assays with at least $1000 \times$ coverage and $1 \%$ sensitivity (details in Supplemental Methods). Groups were compared by chisquare test; TFS was computed from diagnosis to treatment and analyzed by log-rank test and Cox regression analysis with a stepwise procedure using MedCalc Statistical Software version 16.8.4 (MedCalc Software bvba, Ostend, Belgium; https://www.medcalc.org; 2016).

The mutation analysis of the Ras-MAPK pathway was focused on the $K R A S, N R A S$, and $B R A F$ genes, previously reported as the most frequently mutated genes among the members of the pathway [2]. We found 91 missense point mutations in 64 CLL cases, with a prevalence of $K R A S$ (44 mutations in 38 [7.1\%] patients), followed by BRAF (32 mutations in 24 [4.5\%] patients) and NRAS (15 mutations in 13 [2.4\%] patients). Nearly all mutations were previously associated with the gain-of-function phenotype and increased RAS/ERK downstream signaling (Fig. 1a and Table S3) [1]. In particular, among the most frequent KRAS/ NRAS mutations, almost half of the mutations $(27 / 59,45 \%)$, overall affecting $23 / 49$ (47\%) patients, involved the G12/ G13 codons, in keeping with what was observed in colon and lung cancers (Table S3) [1]. The co-occurrence of 2 mutated genes was observed in 11 cases (KRAS and BRAF in 8/11 cases, KRAS and NRAS in 2/11 cases, NRAS and $B R A F$ in $1 / 11$ cases), whereas mutations affecting all three genes were not found in our cohort. The mutations were mainly subclonal (mean Variant Allele Fraction, VAF, $12.3 \%$, range $1.3-61.6 \%$ ) with one-third of mutations (33/ 91) above $10 \% \mathrm{VAF}$. The presence of multiple mutations affecting the same gene occurred in 14 cases, including 5 cases that presented mutations in the same or adjacent codons (i.e., one case with both K601N and K601E BRAF mutations, one case with V600E and K601E BRAF mutations, and three cases with two simultaneous KRAS mutations at the G12 and G13 codons) suggesting that multiple genetic hits are positively selected in different subclones within the same leukemia specimen.

A strong association between KRAS/NRAS/BRAF mutations and the presence of an UM IGHV gene status and trisomy 12 was observed (Fig. 1b and Table S4). Overall, $87.3 \%$ of KRAS/NRAS/BRAF mutated cases had UM IGHV $(p<0.0001)$ and $79.7 \%$ were trisomy 12 CLL $(p<0.0001)$. Concordantly, the highest KRAS/NRAS/BRAF mutation frequency was found in CLL patients with concomitant UM $I G H V$ and trisomy 12 -only $(38 / 133,28.6 \%)$. This group was characterized by 25 KRAS (18.8\%), 8 NRAS (6\%) and 11 BRAF (8.3\%) mutated cases. Of note, the UM IGHVI trisomy 12-only group was characterized by a higher frequency of KRAS/NRAS/BRAF mutations also when compared to the UM $I G H V /$ trisomy 12-plus group $(8 / 49$, $16.3 \%$ ). Finally, the lowest frequency of KRAS/NRAS/ $B R A F$ mutations was observed in the context of CLL patients with $\mathrm{M} I G H V(8 / 186,4.3 \%)$ and del13q as the sole chromosomal aberration $(2 / 94,2.1 \%$ in the whole cohort, and $2 / 53,3.8 \%$ in the context of UM IGHV cases).

We then correlated the presence of KRAS/NRAS/BRAF mutations to other biological features (Table S4). When considering the whole CLL cohort, the only variables associated with a higher frequency of KRAS/NRAS/BRAF mutations were the absence of BIRC3 mutations $(p=0.02)$ and the positive expression $(\geq 30 \%)$ of CD49d $(p=0.04)$. On the other hand, if circumscribing the analysis to UM IGHV/trisomy $12 \mathrm{CLL}, \mathrm{CD} 49 \mathrm{~d}$ positive expression lost its association with $K R A S / N R A S / B R A F$ mutations, as expected due to the almost universal CD49d expression in trisomy 12 CLL patients [9]. Conversely, we observed a higher frequency of KRAS/NRAS/BRAF mutations in NOTCH1 wild type cases $(29 / 92,31.5 \%)$ and $B I R C 3$ wild type cases $(41 / 132,31.1 \%)$ compared to their mutated counterparts (NOTCH1 mutated: 17/90, 18.9\%; BIRC3 mutated: 4/30, $13.3 \% ; p=0.05$ in both cases), pointing to a mutual exclusivity of these mutations in the pathogenesis of the disease. No other significant associations with other known prognostic variables such as presence of TP53 mutations/ disruption, SF3B1 mutations, ZAP-70 and CD38 expression, Rai staging, age at diagnosis, and gender were observed either in the whole cohort or in the UM IGHVI trisomy 12 cohort (Table S4).

We finally evaluated the prognostic relevance of $K R A S, N R A S$, and $B R A F$ mutations as predictors of TFS. In the context of the clinical cohort, the presence of either $K R A S$ or NRAS mutations or the concomitant presence of KRAS/NRAS mutations were associated with shorter TFS ( $p=0.07, p=0.05$, and $p=0.02$, respectively) (Table 1 , Fig. 1c). Conversely, BRAF mutations were not associated with TFS, pointing to a secondary role of BRAF in the Ras-MAPK pathway in CLL, in line with studies indicating the lack of therapeutic effects of BRAF inhibition in CLL [11]. In a multivariable model that included the main known CLL prognosticators, the presence of KRAS/NRAS mutations retained its independent prognostic power as predictor for shorter TFS $(p=0.03$, Table 1). Moreover, circumscribing the analysis to the CLL subgroup with the highest incidence of these mutations (i.e., UM IGHV/trisomy 12-only/NOTCH1wt), both KRAS mutations alone $(p=0.005)$ and KRAS/ 

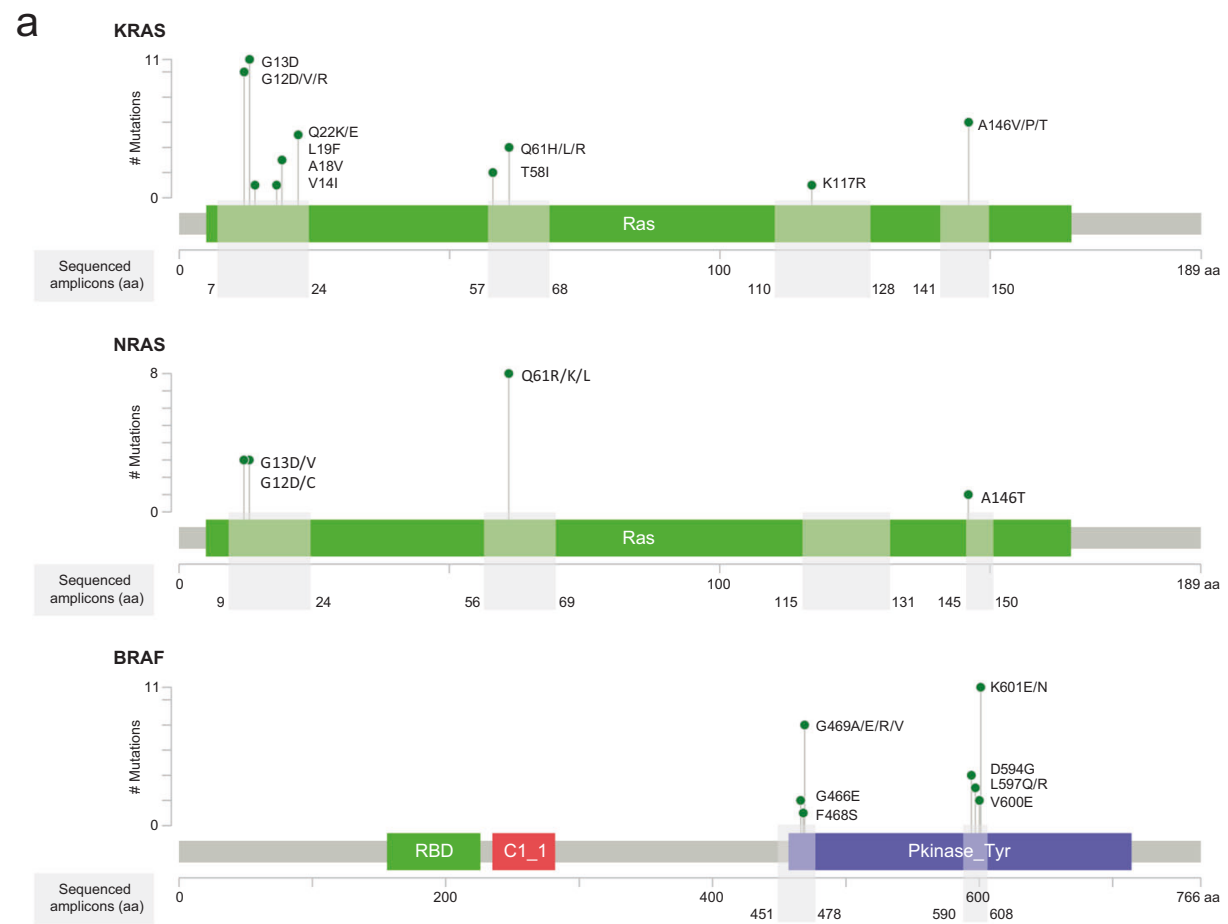

b

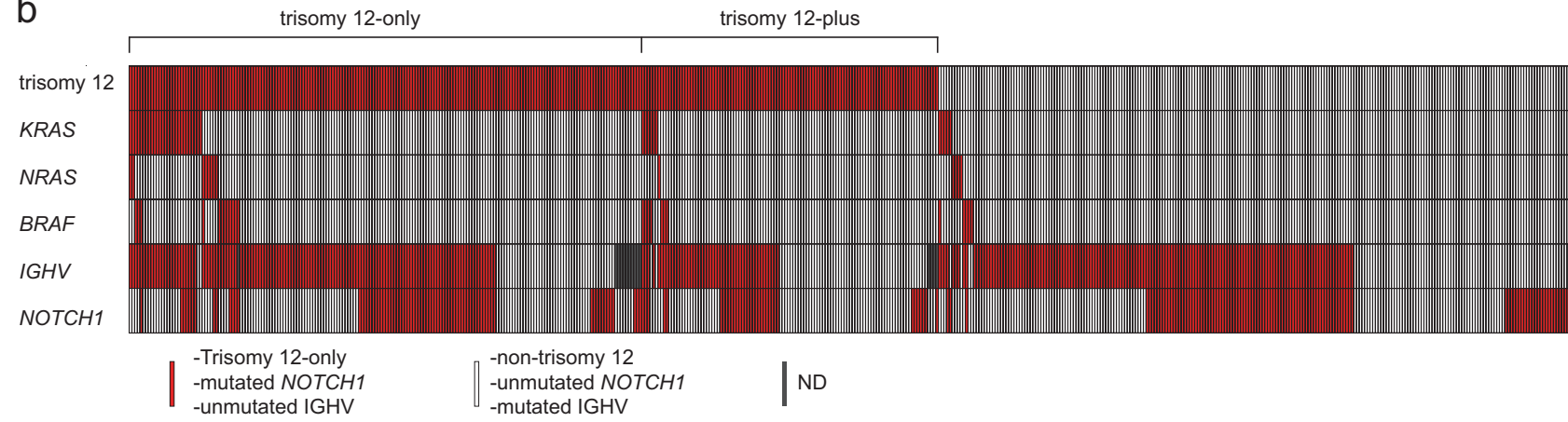

C

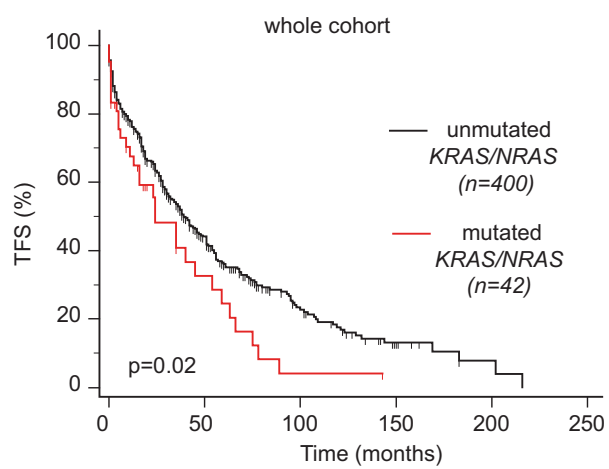

Fig. 1 Type, incidence and prognostic impact of KRAS, NRAS and $B R A F$ mutations. a Lollipop plots of mutations found in $K R A S, N R A S$, and $B R A F$ genes. Sites and frequency of missense point mutations, and schematic presentation of the protein structure and functional domains are shown (MutationMapper, cBioPortal Version 1.14.0, Gao et al. Sci. Signal. 2013 and Cerami et al. Cancer Discov. 2012). Gray boxes indicate amino acids (aa) regions corresponding to the sequenced amplicons. $R B D$, Ras-binding domain; $C 1 \_1$, phorbol esters/diacylglycerol binding domain ( $\mathrm{C} 1$ domain); Pkinase_Tyr, protein

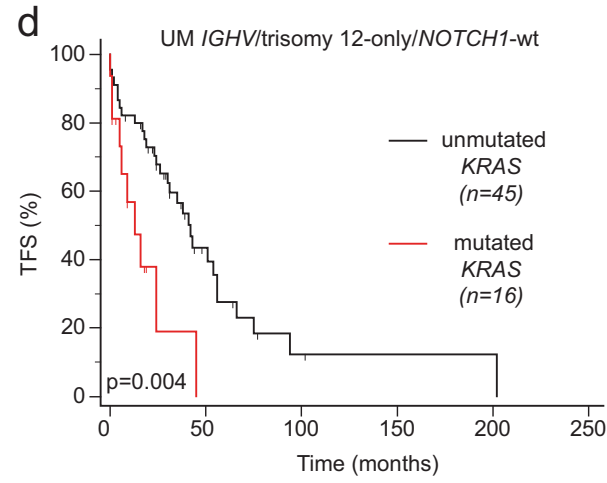

tyrosine kinase domain. b Co-mutation plot of 534 CLL analyzed for $K R A S, N R A S, B R A F$ mutations. Incidence of trisomy 12, KRAS, NRAS, and BRAF missense mutations, NOTCHI aberrations and IGHV status are shown. c Kaplan-Meier curves of treatment-free survival (TFS) of 442 CLL patients stratified by the presence of KRAS and/or NRAS mutations. d Kaplan-Meier curves of TFS of 61 CLL patients in the $I G H V$ unmutated/trisomy 12-only/NOTCHI-wt group stratified by the presence of KRAS mutations 
Table 1 Cox regression analysis of treatment-free survival in the whole cohort

\begin{tabular}{|c|c|c|c|c|c|}
\hline & \multirow[b]{2}{*}{$\begin{array}{l}\mathrm{N} \text { pts } \\
\text { analyzed }\end{array}$} & \multirow{2}{*}{$\begin{array}{l}\text { Univariable } \\
\text { HR (95\% CI) }\end{array}$} & \multirow[b]{2}{*}{$p$-value } & \multicolumn{2}{|c|}{ Multivariable $(n=365)$} \\
\hline & & & & HR $(95 \% \mathrm{CI})$ & $p$-value \\
\hline male gender & 442 & $0.97(0.76-1.22)$ & 0.8 & - & - \\
\hline age $\geq 65$ & 441 & $0.97(0.77-1.23)$ & 0.8 & - & - \\
\hline Rai stage II-III-IV & 434 & $2.60(2.03-3.32)$ & $<0.0001$ & $2.59(1.98-3.41)$ & $<0.0001$ \\
\hline CD49d positive $(\geq 30 \%)$ & 440 & $1.48(1.13-1.92)$ & 0.004 & $1.65(1.22-2.23)$ & 0.001 \\
\hline CD38 positive $(\geq 30 \%)$ & 439 & $1.13(0.89-1.43)$ & 0.3 & - & - \\
\hline ZAP-70 positive $(\geq 20 \%)$ & 389 & $1.44(1.11-1.87)$ & 0.005 & n.i. & n.i. \\
\hline I $G H V$ unmutated & 428 & $2.16(1.66-2.8)$ & $<0.0001$ & $1.83(1.37-2.45)$ & $<0.0001$ \\
\hline $\begin{array}{l}\text { TP53 disrupted (del17p and/or } \\
\text { TP53 mutated) }\end{array}$ & 442 & $1.70(1.24-2.32)$ & 0.0008 & $1.46(1.05-2.03)$ & 0.024 \\
\hline NOTCH1 mutated & 442 & $1.37(1.08-1.73)$ & 0.009 & n.i. & n.i. \\
\hline SF3B1 mutated & 327 & $1.46(0.90-2.38)$ & 0.1 & - & - \\
\hline BIRC3 mutated & 343 & $0.93(0.65-1.34)$ & 0.7 & - & - \\
\hline$K R A S$ mutated & 442 & $1.49(0.96-2.30)$ & 0.073 & - & - \\
\hline NRAS mutated & 442 & $1.86(0.99-3.50)$ & 0.055 & - & - \\
\hline$B R A F$ mutated & 442 & $1.34(0.75-2.40)$ & 0.3 & - & - \\
\hline KRAS/NRAS mutated & 442 & $1.54(1.05-2.25)$ & 0.025 & $1.56(1.04-2.36)$ & 0.033 \\
\hline
\end{tabular}

Factors with $p$-value $<0.05$ in univariable analysis were entered in the multivariable analysis

$H R$ hazard ratio, $C I$ confidence interval, $n . i$. variables not included in the model after stepwise selection
NRAS mutations $(p=0.05)$ were associated with shorter TFS (Fig. 1d and Table S5), and the presence of KRAS mutations retained its prognostic value in a multivariable analysis that included all the variables with an impact in univariable analysis $(p=0.01$, Table S5). The subclonal or clonal pattern of KRAS/NRAS mutations had similar negative impact in our series (not shown), as previously observed for other gene mutations in CLL [12], and in keeping with the known capability of KRAS mutated tumor cells to enhance the overall tumor cell fitness by influencing the non-mutated neoplastic component [13].

In the present study, we demonstrated that KRAS, $N R A S$, and BRAF mutations were almost exclusively found in UM IGHV/trisomy $12 \mathrm{CLL}$ and were almost mutually exclusive with NOTCH1 and BIRC 3 mutations. The type of genomic structural variants, especially trisomy 12 and del13q, strongly influenced KRAS/NRAS/ $B R A F$ mutation incidence, that turned out to be at the highest level in cases bearing trisomy 12 as the sole genomic aberration, intermediate in cases in which trisomy 12 was associated with other genetic aberrations, mainly del13q, and at the lowest level in cases bearing del13q as the sole FISH detectable genetic aberration. This peculiar distribution of KRAS/NRAS/BRAF mutation incidence is in keeping with a CLL pathogenetic model in which the two main founder genetic lesions (i.e., trisomy 12 and del13q) identify CLL subgroups following different patho-biological pathways. In particular, the presence of del13q, given its link to the miR15/miR16-
BCL2 axis, characterizes a CLL subset especially oriented toward the amplification of anti-apoptotic signals [14]. On the other hand, in trisomy 12 CLL, the copresence of KRAS/NRAS/BRAF mutations and/or NOTCH1 mutations and/or BIRC3 mutations along with a UM $I G H V$ gene status and over-expression of surface receptors mediating microenvironment interactions (e.g., CD49d) more likely characterizes CLL with amplified pro-survival and proliferative signals $[8,9,15]$. This may explain the clinical association between KRAS/NRAS mutations and shorter TFS, as shown in the present analysis.

Given the reported high risk of poor response and development of chemo-resistance characterizing CLL cases with KRAS/NRAS mutations [4-6], additional therapeutic strategies should be considered for the treatment of these cases, including MEK/ERK inhibitors, employed alone or in combination with conventional therapies.

Acknowledgements The study was supported by the Fondazione Umberto Veronesi, Post-doctoral Fellowships-year 2018 (to EV); Associazione Italiana Ricerca Cancro (AIRC), Investigator Grant IG21687; Progetto Giovani Ricercatori no. GR-2011-02346826, no. GR2011-02347441, and no.GR-2011-02351370, Ministero della Salute, Rome, Italy; Progetto Ricerca Finalizzata PE 2016-02362756, Ministero della Salute, Rome, Italy; Associazione Italiana contro le Leucemie, linfomi e mielomi (AIL), Venezia Section, Pramaggiore Group, Italy; Linfo-check - Bando ricerca - contributo art. 15, comma 2, lett b) LR 17/2014; "5x1000 Intramural Program", Centro di Riferimento Oncologico, Aviano, Italy; National Cancer Institute, CA197120 (to TDS and NEK). Authors wish to thank Gustavo Baldassarre (Division 
of Molecular Oncology, Department of Translational Research, CRO Aviano, Italy) for helpful discussion.

\section{Compliance with ethical standards}

Conflict of interest The authors declare that they have no conflict of interest.

Publisher's note: Springer Nature remains neutral with regard to jurisdictional claims in published maps and institutional affiliations.

Open Access This article is licensed under a Creative Commons Attribution 4.0 International License, which permits use, sharing, adaptation, distribution and reproduction in any medium or format, as long as you give appropriate credit to the original author(s) and the source, provide a link to the Creative Commons license, and indicate if changes were made. The images or other third party material in this article are included in the article's Creative Commons license, unless indicated otherwise in a credit line to the material. If material is not included in the article's Creative Commons license and your intended use is not permitted by statutory regulation or exceeds the permitted use, you will need to obtain permission directly from the copyright holder. To view a copy of this license, visit http://creativecommons. org/licenses/by/4.0/.

\section{References}

1. Simanshu DK, Nissley DV, McCormick F. RAS proteins and their regulators in human disease. Cell. 2017;170:17-33.

2. Landau DA, Tausch E, Taylor-Weiner AN, Stewart C, Reiter JG, Bahlo J, et al. Mutations driving CLL and their evolution in progression and relapse. Nature. 2015;526:525-30.

3. Puente XS, Bea S, Valdes-Mas R, Villamor N, Gutierrez-Abril J, Martin-Subero JI, et al. Non-coding recurrent mutations in chronic lymphocytic leukaemia. Nature. 2015;526:519-24.

4. Herling CD, Abedpour N, Weiss J, Schmitt A, Jachimowicz RD, Merkel $\mathrm{O}$, et al. Clonal dynamics towards the development of venetoclax resistance in chronic lymphocytic leukemia. Nat Commun. 2018;9:727.

5. Pandzic T, Larsson J, He L, Kundu S, Ban K, Akhtar-Ali M, et al. Transposon mutagenesis reveals fludarabine resistance mechanisms in chronic lymphocytic leukemia. Clin Cancer Res. 2016;22:6217-27.

6. Takahashi K, Hu B, Wang F, Yan Y, Kim E, Vitale C, et al. Clinical implications of cancer gene mutations in patients with chronic lymphocytic leukemia treated with lenalidomide. Blood. 2018;131:1820-32.

7. Gimenez N, Martinez-Trillos A, Montraveta A, Lopez-Guerra M, Rosich L, Nadeu F, et al. Mutations in RAS-BRAF-MAPKERK pathway define a specific subgroup of patients with adverse clinical features and provide new therapeutic options in chronic lymphocytic leukemia. Haematologica. 2018;104: 576-86.

8. Bulian P, Bomben R, Bo MD, Zucchetto A, Rossi FM, Degan M, et al. Mutational status of IGHV is the most reliable prognostic marker in trisomy 12 chronic lymphocytic leukemia. Haematologica. 2017;102:e443-e446.

9. Zucchetto A, Caldana C, Benedetti D, Tissino E, Rossi FM, Hutterer E, et al. CD49d is overexpressed by trisomy 12 chronic lymphocytic leukemia cells: evidence for a methylation-dependent regulation mechanism. Blood. 2013;122:3317-21.

10. Hallek M, Cheson BD, Catovsky D, Caligaris-Cappio F, Dighiero $\mathrm{G}$, Dohner $\mathrm{H}$, et al. iwCLL guidelines for diagnosis, indications for treatment, response assessment, and supportive management of CLL. Blood. 2018;131:2745-60.

11. Yaktapour N, Meiss F, Mastroianni J, Zenz T, Andrlova H, Mathew NR, et al. BRAF inhibitor-associated ERK activation drives development of chronic lymphocytic leukemia. J Clin Invest. 2014;124:5074-84.

12. Nadeu F, Clot G, Delgado J, Martin-Garcia D, Baumann T, Salaverria I, et al. Clinical impact of the subclonal architecture and mutational complexity in chronic lymphocytic leukemia. Leukemia. 2018;32:645-53.

13. Grabocka E, Bar-Sagi D. Mutant KRAS enhances tumor cell fitness by upregulating stress granules. Cell. 2016;167:1803-13.

14. Cimmino A, Calin GA, Fabbri M, Iorio MV, Ferracin M, Shimizu M, et al. miR-15 and miR-16 induce apoptosis by targeting BCL2. Proc Natl Acad Sci USA. 2005;102:13944-9.

15. Benedetti D, Tissino E, Pozzo F, Bittolo T, Caldana C, Perini C, et al. NOTCH1 mutations are associated with high CD49d expression in chronic lymphocytic leukemia: link between the NOTCH1 and the NF-kappaB pathways. Leukemia. 2018;32: 654-62. 